\title{
AN EQUIVARIANT WALL OBSTRUCTION THEORY
}

\author{
BY
}

JENNY A. BAGLIVO

\begin{abstract}
Let $G$ be a finite group. For a certain class of $C W$-complexes with a $G$-action which are equivariantly dominated by a finite complex we define algebraic invariants to decide when the space is equivariantly homotopy or homology equivalent to a finite complex.
\end{abstract}

1. Introduction. A connected CW-complex $X$ is a $G$-complex if the group $G$ acts on $X$ as a group of homeomorphisms which permute the cells of $X$. The question we study here is: Suppose that $G$ is a finite group, that $X$ is a $G$-complex and that $K$ is a finite $G$-complex. Suppose there exist equivariant cellular maps $\varphi: K \rightarrow X$ and $s: X \rightarrow K$ and an equivariant cellular homotopy $\varphi s \simeq 1_{X}$. (K G-dominates $X$.) Is $X$ "equivalent" to a finite $G$-complex?

Our main result is the following theorem.

THEOREM (5.1). Let $G$ be a finite group. Let $X$ be a $G$-complex such that for each subgroup $H$ of $G, X^{H}$ is connected, and such that $X^{G} \neq \varnothing$. Suppose that $X$ is $G$-dominated by a finite $G$-complex. Then

(i) there exists an invariant $E_{1}(X, G)$ such that $X$ is $G$-equivalent to a finite $G$-complex if and only if $E_{1}(X, G)$ vanishes;

(ii) there exists an invariant $E_{2}(X, G)$ such that $X$ is $G$-homology-equivalent to a finite G-complex where the equivalence is G-2-connected if and only if $E_{2}(X, G)$ vanishes.

The space $X^{H}$ is the subcomplex of $X$ fixed by the subgroup $H$. A $G$-equivalence is an equivariant homotopy equivalence. As the term suggests, a $G$-homology-equivalence is an algebraic concept. (See \$5.) The notions are not equivalent but each is a homotopy equivalence.

The elements $E_{1}(X, G)$ and $E_{2}(X, G)$ are Wall-type invariants [15], [16] of equivariant homotopy type. They are defined by considering chain complexes related to the cellular chains of the universal cover of $X, C(\tilde{X})$. (See $\$ 5$.

In $\$ 2$ we discuss lifting group actions to regular covers of a $G$-complex $X$. $\$ 3$ then shows how to factor $C(\tilde{X})$ as needed for the main theorem. Equivariant cell-attaching is discussed in $\$ 4$, where we also show that if $X$ is $G$-dominated by a finite $G$-complex, then it is $G$-equivalent to a complex of

Presented to the Society, January 29, 1977; received by the editors May 31, 1977.

AMS (MOS) subject classifications (1970). Primary 55G99, 57E99, 54E60.

Key words and phrases. G-complex, Wall obstruction, G-equivalence, G-homology equivalence.

() 1979 American Mathematical Society 
finite type. $\S 5$ is devoted to a proof of Theorem (5.1). Lastly, in $\S 6$, we study the notion of $G$-homology-equivalence further. The results we obtain here are more algebraic in flavor than in the rest of the paper.

Most of this work is the author's thesis, written under the direction of Professor Douglas Anderson of Syracuse University. I wish to thank him for suggesting the problem to me, and for his advice and support.

2. Definition of $G$-complex; lifting group actions. Let $X$ be a connected CW-complex with fixed cellular decomposition and let $C(X)$ denote the group of cellular isomorphisms $f$ of $X$ with the property that if $f$ leaves a cell of $X$ invariant, then $f$ fixes the cell pointwise. Let $G$ be a group which we write multiplicatively.

Definition 1. A cellular action of $G$ on $X$ is a homomorphism $\alpha$ : $G \rightarrow$ $\mathcal{C}(X)$, denoted by $\alpha(g) \equiv g$. The space $X$ with a fixed cellular action of $G$ is called a $G$-complex. The action is said to be effective if ker $\alpha=\{1\}$.

Whenever possible we suppress mentioning the homomorphism $\alpha$. If $H$ is a subgroup of $G$ and $X$ is a $G$-complex, then $X$ is an $H$-complex by simply restricting the homomorphism $\alpha$ to $H$.

Definition 2. Let $X$ and $Y$ be $G$-complexes. A map $\varphi: X \rightarrow Y$ is said to be a $G$-map if $\varphi$ is a cellular map which respects the action of $G$, that is, such that $\varphi(g x)=g \varphi(x), x \in X$ and $g \in G$.

We follow the notation and terminology of [2]. Thus, in particular, if $S \subseteq G$ we let $X^{S}$ denote the fixed-point set of $S$ in $X$.

In Lemma 3 we list some facts which follow easily from the definitions. If $H$ is a subgroup of $G$ we let $N H$ denote the normalizer of $H$ in $G$. We use the notation $H \leqslant G$ if $H$ is a subgroup of $G$, and $H<G$ if $H$ is a proper subgroup of $G$.

LEMMA 3. Let $X$ be a $G$-complex and let $H$ be a subgroup of $G$.

(i) The subspace $X^{H}$ is $N H$-invariant. In particular, if $H$ is normal in $G$ then $X^{H}$ is invariant under the action of $G$.

(ii) There is an induced action of $N H / H$ on $X^{H}$. Thus $X^{H}$ is also an NH/H-complex.

(iii) The restriction of $g$ to $X^{H}$ yields a homeomorphism $g: X^{H} \rightarrow X^{g^{H g} g^{-1}}$, for each $g \in G$.

(iv) Let $X^{>H}=\cup_{H^{\prime}>H} X^{H^{\prime}}$. Then $X^{>H}$ is an NH-complex, and an $\mathrm{NH} / \mathrm{H}$-complex.

We will be interested in considering group actions defined on a regular cover of $X, \hat{X}$, which lift the action of $G$ on $X$. If an action exists the group involved is, in general, an extension of $G / \operatorname{ker} \alpha$ by the group of deck transformations of the cover, and it is a split extension of these groups if there is a fixed point of $G$ in $X$. (See [2].) For technical reasons we will include here 
actions which are not effective. If $G$ and $\pi$ are groups, we use the notation $\pi x_{\alpha} G$ to represent the semidirect product of $\pi$ and $G$ with respect to an action $\alpha: G \rightarrow \mathbb{Q}(\pi)$, where $\alpha$ is a homomorphism whsoe range is the group of automorphisms of $\pi$.

Let $X$ be a connected $G$-complex and let $e^{0} \in X^{G}$. Then $G$ acts on $\pi_{1}\left(X, e^{0}\right)$ by setting $g \cdot \gamma=\pi_{1}(g)(\gamma)$. We denote this action by $\alpha$. Let $p$ : $\hat{X} \rightarrow X$ be a regular cover of $X$. If $\operatorname{im}\left(\pi_{1}(p)\right)$ is invariant under the action of $G$, then there is an induced action (also denoted by $\alpha$ ) on $\pi_{1}\left(X, e^{0}\right) / \operatorname{im}\left(\pi_{1}(p)\right)$. Let $\hat{G}=\pi_{1}\left(X, e^{0}\right) / \operatorname{im}\left(\pi_{1}(p)\right) x_{\alpha} G$.

LEMMA 4. In the situation described above, the space $\hat{X}$ is a $\hat{G}$-complex.

Proof. Fix a point $\hat{e}^{0} \in \hat{X}$ over $e^{0}$ and use the Lifting Map Theorem to construct $\hat{g}:\left(\hat{X}, \hat{e}^{0}\right) \rightarrow\left(\hat{X}, \hat{e}^{0}\right)$ such that $p \hat{g}=g p$, for $g \in G$.

The action of $\hat{G}$ on $\hat{X}$ is defined by $(\gamma, g) x \equiv \gamma(\hat{g}(x)), \gamma \in$ $\pi_{1}\left(X, e^{0}\right) / \operatorname{im}\left(\pi_{1}(p)\right), g \in G, x \in \hat{X}$.

Notice that the action of $G$ depends on the choices of $e^{0} \in X^{G}$ and $\hat{e}^{0} \in \hat{X}$.

As a special case of Lemma 4 , the universal cover of $X, \tilde{X}$, is a $\tilde{G}$-complex, with $\tilde{G}=\pi_{1}(X) x_{\alpha} G$. If $A \subset X$ is a connected subcomplex, then there is a regular cover $\hat{A}$ of $A$ in $\tilde{X}$. We next discuss extensions of actions to $\hat{A}$.

LEMMA 5. Let $X$ be a connected $G$-complex such that $X^{G} \neq \varnothing$. Let $A \subset X$ be a connected G-invariant subcomplex such that $A^{G} \neq \varnothing$. Let $\hat{A} \subset \tilde{X}$ be one component of $p^{-1}(A)$.

(i) The space $A$ with regular cover $\left.p\right|_{A}: \hat{A} \rightarrow A$ satisfies Lemma 4. Thus, $\hat{A}$ is a $\hat{G}$-complex where $\hat{G}=\pi_{1}(A) / \operatorname{im}\left(\pi_{1}\left(\left.p\right|_{A}\right)\right) x_{\alpha} G$.

(ii) Let $\tilde{G}=\pi_{1}(X) x_{\alpha} G$. Then $\hat{G}$ is a subgroup of $\tilde{G}$ and $\hat{A}$ is a $\hat{G}$-invariant subspace of $\tilde{X}$.

Proof. (i) We need to show that $\operatorname{im}\left(\pi_{1}\left(\left.p\right|_{A}\right)\right) \subset \pi_{1}(A)$ is $G$-invariant. (We assume that the basepoint is some $e^{0} \in A^{G}$.) $\operatorname{But} \operatorname{im}\left(\pi_{1}\left(\left.p\right|_{A}\right)\right)=\operatorname{ker}\left(\pi_{1}(i)\right)$, where $i: A \hookrightarrow X$ is the inclusion map. Since $A$ is $G$-invariant, $i$ is a $G$-map. The group $\operatorname{ker}\left(\pi_{1}(i)\right)$ is, therefore, a $G$-invariant subgroup.

(ii) We assume that the basepoints for the fundamental groups are $e^{0} \in A^{G}$ $\subset X^{G}$ and $\hat{e}^{0} \in \hat{A} \subset \tilde{X}$. Then

$$
\pi_{1}(A) / \operatorname{im}\left(\pi_{1}\left(\left.p\right|_{A}\right)\right)=\pi_{1}(A) / \operatorname{ker}\left(\pi_{1}(i)\right) \cong \operatorname{im}\left(\pi_{1}(i)\right) \leqslant \pi_{1}(X) ;
$$

and thus $\hat{G} \leqslant \tilde{G}$ under this identification. The rest of the lemma follows from the construction in Lemma 4.

In particular we can apply Lemma 5 to fixed-point sets of a subgroup $H$ of $G$ in $X$. Suppose that $X^{H}$ and $X^{>H}=\cup_{H^{\prime}>H} X^{H^{\prime}}$ are connected. These complexes are $N H$-invariant, and thus by Lemma $5, X^{H}$ and $X^{>H}$ are $N H$-complexes which are $N H$-invariant subcomplexes of $\tilde{X}$. 
3. Permutation complexes; the structure of the cellular chain complex $C(\tilde{X})$. Let $X$ be a connected $G$-complex and $p: \tilde{X} \rightarrow X$ be its universal cover. Suppose that $X^{G} \neq \varnothing$. By the discussion in $\S 2, \tilde{X}$ is a $\tilde{G}$-complex, where $\tilde{G}=\pi_{1}(X) x_{\alpha} G$. Then the cellular chain complex $C(\tilde{X})$ is a complex over the group ring $\mathbf{Z} \tilde{G}$. The purpose of this section is to study the structure of this chain complex further. In particular, we prove the following proposition.

Proposition 1. Let $X$ be a connected $G$-complex such that for every subgroup $H$ of $G, X^{H}$ is connected. Suppose also that $X^{G} \neq \varnothing$. Then the following statements hold:

(i) The complex $C(\tilde{X})$ is a $\mathbf{Z} \tilde{G}$-permutation complex.

(ii) Let $\tilde{X}^{H}$ represent the universal cover of $X^{H}$. The subcomplex

$$
C\left(p^{-1}\left(X^{H}\right)\right) \approx \mathrm{Z}\left(\pi_{1}(X) x_{\alpha} N H\right) \underset{\mathrm{Z}\left(\pi_{1}\left(X^{H}\right) x_{\alpha} N H\right)}{\otimes} C\left(\tilde{X}^{H}\right),
$$

as complexes of $\mathbf{Z}\left(\pi_{1}(X) x_{\alpha} N H\right)$-modules.

We will first state the relevant definitions, facts and conventions. Recall that if $S$ is a set and $\mathscr{B}(S)$ is the group of bijections of $S$, an action of $G$ on $S$ as a group of permutations is a homomorphism $\alpha: G \rightarrow \mathscr{B}(S)$. We use the usual notation of $G_{x}$ for the isotropy group of a point $x$, and $G(x)$ for its orbit. Let $R$ be a ring with unit.

Definition 2. An $R G$-permutation module, $M$, is a left $R G$-module which is free as an $R$-module with a distinguished basis $S$ on which $G$ acts as a group of permutations, and whose $R G$-module structure extends this action on $S$ linearly. We distinguish several $R$-free submodules of $M$, with $H \leqslant G$ a subgroup:

$M^{H}$ is $R$-free with basis $S^{H}=\left\{x \in S: G_{x} \geqslant H\right\}$;

$M^{>H}$ is $R$-free with basis $S^{>H}=\left\{x \in S: G_{x}>H\right\}$;

$M_{H}$ is $R$-free with basis $S_{H}=\left\{x \in S: G_{x}=H\right\}$.

If the distinguished basis of $M$ is $G(x)$, the orbit of one element $x \in S$, we can identify $G(x)$ with $G / H$, the set of cosets of $H=G_{x}$, with action defined by left multiplication. This identification depends on the choice of $x \in S$. However, once a choice is made, we can write $M=R(G / H)$. In fact, all $R G$-permutation modules are $R G$-direct sums of such modules. We can write $M=\Sigma_{H<G} \oplus_{R} M_{H}=\cup_{H<G} M^{H}$.

As an $R$-module $M^{H}$ decomposes into $M^{>H} \oplus M_{H}$. The modules $M^{H}, M_{H}, M^{>H}$ are not in general $R G$-invariant submodules of $M$. They are, however, invariant submodules of $M$ under the action of the normalizer of $H$ in $G$.

LEMMA 3. Let $M$ be an RG-permutation module.

(i) The modules $M^{H}, M_{H}$ and $M^{>H}$ are $R(N H)$-and $R(N H / H)$ permutation modules. The module $M_{H}$ is a free $R(N H / H)$-module; 
(ii) The modules $\cup_{g \in G} M^{g H_{g}^{-1}}, \cup_{g \in G} M_{g g^{-1}}$ and $\cup_{g \in G} M^{>g H^{-1}}$ are $R G$-invariant submodules of $M$;

(iii) For each $g \in G$, the action of $g$ induces an $R$-isomorphism $g: M^{H} \rightarrow$ $M^{g g^{-1}}$, so that $g\left(M_{H}\right)=M_{g g^{-1}}$ and $g\left(M^{>H}\right)=M^{>H_{g}^{-1}}$;

(iv) For each $g \in G$, the group isomorphism $\mathrm{g}: \mathrm{NH} \rightarrow \mathrm{NgHg}^{-1}$ defined by $k \rightarrow g \mathrm{~kg}^{-1}$ extends linearly to an isomorphism of the group rings. We can represent

$$
M^{g H g^{-1}} \cong R\left(N g H g^{-1}\right) \underset{R(N H)}{\bigotimes} M^{H}
$$

and we can represent $M_{\mathrm{gHg}^{-1}}$ and $\mathrm{M}^{>\mathrm{gHg}^{-1}}$ similarly.

(v) For each $\mathrm{g} \in \mathrm{G}$, the group isomorphism $\mathrm{g}: \mathrm{NH} / \mathrm{H} \rightarrow \mathrm{NgHg}$ induced from the isomorphism in (iv) extends linearly to an isomorphism of the group rings. We can represent

$$
M^{\mathrm{gHg}^{-1}} \simeq R\left(\mathrm{NgHg}^{-1} / \mathrm{gHg}^{-1}\right) \underset{R(\mathrm{NH} / \mathrm{H})}{\otimes} M^{H},
$$

and we can represent the other distinguished submodules similarly.

Proof. All parts are straightforward applications of the definitions.

Definition 4. Let $M$ and $M^{\prime}$ be $R G$-permutation modules. An $R G$-homomorphism $\varphi: M \rightarrow M^{\prime}$ is said to be an $R G$-permuatation homomorphism if $\operatorname{im}\left(\left.\varphi\right|_{M^{H}}\right) \subset M^{\prime H}$ for each subgroup $H$ of $G$. We let $\varphi^{H}$ denote $\left.\varphi\right|_{M^{H}}$, $\varphi^{>H}=\left.\varphi\right|_{M>H}: M^{>H} \rightarrow M^{\prime>H}$ and $\left(\varphi_{H}, \varphi_{H}^{{ }^{H}}\right): M_{H} \rightarrow M_{H}^{\prime} \oplus M^{\prime>H}=M^{\prime H}$.

The reader should note that $R G$-homomorphisms are not in general $R G$ permutation homomorphisms. As an example of this, consider the trivial permutation module $R$ and the free $R G$-module $R G$. Let $G$ be a finite group. Define an $R$-homomorphism $h: R \rightarrow R G$ by $1 \mapsto \sigma=\Sigma_{g \in G} g$. Since $g^{\prime} \sigma=\sigma$ for every $g^{\prime} \in G, h$ is also an $R G$-homomorphism. Now, $h\left(R^{G}\right)=h(R)=$ $R\{\sigma\} \not \subset(R G)^{G}=0$.

The following facts are easily checked using the definitions and Lemma 3.

LEMMA 5. Let $\varphi: M \rightarrow M^{\prime}$ be an RG-permutation homomorphism.

(i) The homomorphisms $\varphi^{H}, \varphi^{>H}$ and $\varphi_{H}$ are $\dot{R}(N H)$ - and $R(N H / H)$-permutation homomorphisms.

(ii) We can represent $\varphi^{g \mathrm{Hg}^{-1}}=R\left(\mathrm{NgHg}^{-1} / \mathrm{gHg}^{-1}\right) \otimes_{R(\mathrm{NH} / \mathrm{H})} \varphi^{H}$, and we can represent $\varphi^{>\mathrm{gHg}^{-1}}$ and $\varphi_{\mathrm{gHg}}{ }^{-1}$ similarly.

(iii) We can represent $\varphi^{g H g}=R\left(\mathrm{NgHg}^{-1}\right) \otimes_{R(\mathrm{NH})} \varphi^{H}$, and we can represent $\varphi^{>\mathrm{gHg}^{-1}}$ and $\varphi_{\mathrm{gHg}}{ }^{-1}$ similarly.

Definition 6. An $R G$-chain complex $C$ is an $R G$-permutation complex if each $C_{i}$ and $\partial_{i}$ is an $R G$-permutation module and homomorphism, respectively. If $\varphi: D \rightarrow C$ is an $R G$-chain map between permutation complexes and each $\varphi_{i}$ is a permutation homomorphism, $\varphi$ is an $R G$-permutation chain map. 
The $R$-subcomplexes $C^{H}, C^{>H}$, and quotient $C_{H}$ are defined in the obvious way and are clearly seen to be $R(N H)$-and $R(N H / H)$-permutation complexes. These complexes satisfy the short exact sequence

$$
C^{>H_{2}} \rightarrow C^{H} \rightarrow C_{H},
$$

but $C^{H}$ is not, in general, a direct sum of the subcomplexes $C^{>H}$ and $C_{H}$ since $\partial^{H}$ is not, in general, a direct sum of homomorphisms.

The proof of Proposition 1 will follow from Lemmas 7-9. Since the proofs of these lemmas are straightforward they will be omitted.

LeMma 7. Let $X$ be a G-complex. The cellular chain complex $C(X)$ is a $\mathrm{ZG}$-permutation complex.

Notice that, as subcomplexes of $C(X), C(X)^{H}=C\left(X^{H}\right)$ and

$$
C(X)_{H}=C\left(X^{H}, \bigcup_{H^{\prime}>H} X^{H^{\prime}}\right)=C\left(X^{H}, X^{>H}\right) .
$$

LeMMA 8. Let $X$ be a connected G-complex such that $e^{0} \in X^{G}$. Let $p: \hat{X} \rightarrow X$ be a regular cover such that $\operatorname{im}\left(\pi_{1}(p)\right) \subset \pi_{1}\left(X, e^{0}\right)$ is invariant under the action of $G$. Let $\hat{G}=\pi_{1}(X) / \operatorname{im}\left(\pi_{1}(p)\right) x_{\alpha} G$.

Then $C(X) \cong \mathbf{Z G} \otimes_{\mathrm{Z} \hat{G}} C(\hat{X})$ as $\mathbf{Z} G$-complexes.

Lemma 9. Let $X$ be a connected $G$-complex such that $X^{G} \neq \varnothing$ and let $p$ : $\tilde{X} \rightarrow X$ be its universal cover. Let $A$ be a connected $G$-invariant subcomplex of $X$ such that $A^{G} \neq \varnothing$ and let $\hat{A} \subset \tilde{X}$ be a regular cover. Let $\tilde{G}=\pi_{1}(X) x_{\alpha} G$ and $\hat{G}=\operatorname{im} \pi_{1}(i) x_{\alpha} G$. Then there is an isomorphism of the chain complexes $C\left(p^{-1}(A)\right) \simeq \mathbf{Z} \tilde{G} \otimes_{\mathbf{Z} \hat{G}} C(\hat{A})$ as $\mathbf{Z} \tilde{G}$-complexes.

Proof of Proposition 1. The first part follows from (2.4) and Lemma 7.

The given representation of $C\left(p^{-1}\left(X^{H}\right)\right)$ follows from Lemmas 8 and 9 above.

4. $G$-equivalence and $G$-domination. Our objectives in this section are to show how to extend the " $G$-connectivity" of a $G$-domination map by an equivariant cell-attaching technique (Lemma 5), and to prove

Proposrrion 1. Let $X$ be a G-complex and $G$ a finite group. If $X$ is $G$-dominated by a finite complex then $X$ is G-equivalent to a complex of finite type, $Y$. If $n$ is the dimension of the dominating complex, then the complex $Y$ is G-dominated by its $n$-skeleton.

These results generalize the constructions in [15]. Note that a complex of finite type is one containing a finite number of cells in each dimension.

If $\varphi$ is a $G$-map and $H \leqslant G$ is a subgroup, we let $\varphi^{H}$ denote the restriction $\left.\varphi\right|_{K^{H}}: K^{H} \rightarrow X^{H}$ and let $M\left(\varphi^{H}\right)$ denote its mapping cylinder. The following definitions will be needed. 
DefinItion 2. A $G$-map $\varphi: K \rightarrow X$ is said to be $G$-n-connected if $\pi_{i}\left(M\left(\varphi^{H}\right), K^{H}\right)=0$ for all $i \leqslant n$, for all $H \leqslant G$, and for all choices of basepoint.

Definition 3. A $G$-map $\varphi: K \rightarrow X$ is said to be a $G$-domination if there exists a $G$-map $s: X \rightarrow K$ and $G$-homotopy $F_{1}: \varphi s \simeq 1_{X}$. In addition, if there exists a $G$-homotopy $F_{2}: s \varphi \simeq 1_{K}, \varphi$ is said to be a $G$-equivalence, and $K$ and $X$ are said to be of the same $G$-homotopy-type.

If $X$ is a $G$-complex, $X \times I$ is a $G$-complex by defining $g(x, t) \equiv(g x, t)$, $x \in X, t \in I$. Thus, a $G$-homotopy $F: f_{0} \simeq f_{1}$ between $G$-maps $f_{0}, f_{1}: X \rightarrow Y$ is a $G$-map $F: X \times I \rightarrow Y$ such that

$$
F(x, i)=f_{i}(x), \quad i=0,1 .
$$

Also, if $\varphi: K \rightarrow X$ is a $G$-map there is an induced action of $G$ on the mapping cylinder $M(\varphi)$, and this action has the property that $M(\varphi)^{H}=M\left(\varphi^{H}\right)$.

From the definitions it is clear that if $\varphi$ is a $G$-equivalence then $\varphi^{H}$ is a homotopy equivalence for each $H$. Thus, $\varphi$ is $G$-n-connected for every $n$. The converse, which is an equivariant version of a theorem of Whitehead, is also true.

Proposition 4. Let $\varphi: K \rightarrow X$ be a G-map. If $\varphi$ is G-n-connected for every $n$ then $\varphi$ is a G-equivalence.

Proof. See [10].

We assume from now on that $G$ is a finite group. Assume that $X$ is $G$-dominated by a finite complex $K$, with $G$-dominating map $\varphi: K \rightarrow X$, and right inverse $s: X \rightarrow K$; and that both $K$ and $X$ are connected. By assumption, $\varphi^{H}: K^{H} \rightarrow X^{H}$ is a dominating map for each $H \leqslant G$. Although $K^{H}$ and $X^{H}$ may have more than one component each, since $K$ is a finite complex both have a finite number of components.

We show that we can increase the $G$-connectivity of the map by successively adding a finite number of cells in each dimension. In the following lemma, let any $G$-dominating map be " $G-(-1)$-connected".

LEMMA 5. Let $X$ be $G$-dominated by the finite complex $K$, with dominating map $\varphi: K \rightarrow X$. Suppose $\varphi$ is $G-(i-1)$-connected. Then there exists a finite $G$-complex $L$ containing $K$ and a G-i-connected dominating map $\psi: L \rightarrow X$ extending $\varphi$.

Proof. If $\psi: L \rightarrow X$ extends $\varphi$, and we let $j: K \rightarrow L$ represent the inclusion, then $j s$ is a homotopy right inverse of $\psi$. Thus, it is sufficient to construct a $G$ - $i$-connected extension of $\varphi$.

To do this we proceed by induction on the order $|H|$ of the subgroup $H$ of $G$. To simplify notation, assume that $\varphi: K \rightarrow X$ is $G-(i-1)$-connected, and $\varphi^{H^{\prime}}: K^{H^{\prime}} \rightarrow X^{H^{\prime}}$ is $i$-connected for all $H^{\prime}$ where $\left|H^{\prime}\right|>|H|$. In the induction 
steps below, we add cells to $K^{H}$ and to $K^{g H g^{-1}}$ for all conjugates to extend the connectivity of $\varphi^{H}$ and all $\varphi^{g \mathrm{Hg}^{-1}}$. There are three cases to consider.

Case $1(i=0)$. By the domination $\pi_{0}\left(\varphi^{H}\right): \pi_{0}\left(K^{H}\right) \rightarrow \pi_{0}\left(X^{H}\right)$ is an epimorphism. If $\varphi^{H}$ is one-to-one on components then it is already 0-connected and there is nothing to do. Suppose not, and suppose components $K_{1}^{H}$ and $K_{2}^{H}$ map to $X_{0}^{H}$, that is, $\varphi^{H}: K_{1}^{H} \cup K_{2}^{H} \rightarrow X_{0}^{H}$. Let $e_{1}, e_{2}$ be zero-cells in $K_{1}^{H}$ and $K_{2}^{H}$ respectively. Let $\omega: I \rightarrow X_{0}^{H}$ be a path from $\varphi^{H}\left(e_{1}\right)$ to $\varphi^{H}\left(e_{2}\right)$ along the one-skeleton of $X_{0}^{H}$. For each class $g \in G / H$ attach a one-cell $e_{g}$ so that the characteristic map on the boundary $c_{g}: \dot{I} \rightarrow K^{g H_{g}^{-1}}$ is $c_{g}(0)=g e_{1}$ and $c_{g}(1)=$ $\mathrm{ge}_{2}$. (This definition is independent of choice of representative for the class in $G / H$ since $H$ fixes $K_{1}^{H}$ and $K_{2}^{H}$.) The cells are permuted by $g^{\prime} e_{g}=e_{g^{\prime} g}$. To extend the map $\varphi$ to $\psi$ define $\left.\psi_{0}^{H}\right|_{g}=g \omega c_{g}^{-1}$.

Case $2(i=1)$. Consider $\varphi^{H}: K^{H} \rightarrow X^{H}$. Since $\varphi$ is 0 -connected $K^{H}$ and $X^{H}$ have the same number of components. Let $\varphi_{0}^{H}: K_{0}^{H} \rightarrow X_{0}^{H}$ be the restriction to corresponding components. Now since $\varphi_{0}^{H}$ is a domination there exists a short exact sequence

$$
1 \rightarrow \pi_{1}\left(M\left(\varphi_{0}^{H}\right), K_{0}^{H}\right) \rightarrow \pi_{1}\left(K_{0}^{H}\right) \rightarrow \pi_{1}\left(X_{0}^{H}\right) \rightarrow 1 .
$$

Also, $\pi_{1}\left(X_{0}^{H}\right)$ is finitely presented, and $\pi_{1}\left(M\left(\varphi_{0}^{H}\right), K_{0}^{H}\right)$ is finitely normally generated in $\pi_{1}\left(K_{0}^{H}\right)$ [15, Proof of Lemma 1.3]. Let $\{\alpha\}$ be a finite set of generators for $\pi_{2}\left(M\left(\varphi_{0}^{H}\right), K_{0}^{H}\right)$. Let $\alpha=\left\langle\left(\beta_{\alpha}, \gamma_{\alpha}\right)\right\rangle$ where

$$
\left(\beta_{\alpha}, \gamma_{\alpha}\right):\left(D^{2}, S^{1}\right) \rightarrow\left(M\left(\varphi_{0}^{H}\right), K_{0}^{H}\right) \text {. }
$$

We attach 2-cells $e_{(g, \alpha)}$ to $K$ via $g \circ \gamma_{\alpha}: S^{1} \rightarrow K^{g H_{g}-1}$. To extend the action over the cells define $g^{\prime} e_{(g, \alpha)} \equiv e_{(g g, \alpha)}$. To extend the map $\varphi$ let $\left.\psi^{H}\right|_{e_{(g, \alpha)}}=$ $g \circ \beta_{\alpha}$.

Case $3(i>1)$. By [15, Proof of Theorem A] $\pi_{i}\left(M\left(\varphi_{0}^{H}\right), K_{0}^{H}\right)$ is a finitelygenerated $\mathbf{Z} \pi_{1}\left(K_{0}^{H}\right)=\mathbf{Z}\left(\pi_{1}\left(X_{0}^{H}\right)\right)$-module. Let $\{\alpha\}$ be a finite set of $\mathbf{Z} \pi_{1}\left(X_{0}^{H}\right)$-generators and proceed as in Case 2.

ProOf OF Proposimon 1. Let $\varphi: K \rightarrow X$ be a $G$-dominating map with right homotopy inverse $s$. Using Lemma 5 inductively we can assume that $\varphi$ is $G-n$-connected, where $n$ is the dimension of $K$. By continuing the construction of Lemma 5 and by Proposition 4 we obtain a $G$-equivalence $\psi$ : $Y \rightarrow X$, where $Y$ is a $G$-complex of finite type and the $n$-skeleton of $Y$ is $K$. Let $s^{\prime}$ be a homotopy inverse of $\psi$. Then the inclusion $K \hookrightarrow Y$ is a $G$ domination with right homotopy inverse $s^{\prime} \varphi s \psi$. (Since all maps are cellular the image of $s^{\prime} \varphi s \psi$ lies in $Y^{n}=K$.)

5. The two $G$-homotopy type invariants. In this section we prove Theorem (5.1), as stated in the introduction.

The definitions of the invariants $E_{1}(X, G)$ and $E_{2}(X, G)$ will require a description of some relative Wall invariants. We first describe briefly the Wall invariant as it will be needed below. See also [15], [16], [6], and [12]. Let $R$ be 
a ring with unit, and let $\tilde{K}_{0}(R)$ be the reduced projective class group of $R$. Let $C$ be an $R$-free chain complex such that $C_{i}$ is finitely-generated (f.g.) for each $i$. Let $C^{(n)}$ be the $n$-skeleton of $C$. If the inclusion $C^{(n)} \hookrightarrow C$ is an $R$-domination of $C$ then

$$
H\left(C, C^{(n)}\right)=H_{n+1}\left(C, C^{(n)}\right)
$$

is a f.g. projective $R$-module. The Wall invariant of $C$ is defined by

$$
w(C)=(-1)^{n+1}\left[H_{n+1}\left(C, C^{(n)}\right)\right] \in \tilde{K}_{0}(R) .
$$

This invariant vanishes if and only if $C$ is $R$-chain equivalent to a finite $R$-free complex.

Now let $X$ satisfy the hypotheses of Theorem 1. Let $\varphi: K \rightarrow X$ be a $G$-dominating map where $K$ is a finite $G$-complex. By (4.5) and (4.1) we may assume that $X$ is of finite-type, and that the inclusion $K \hookrightarrow X$, where $K=X^{n}$ and $n \geqslant 2$, is a $G$-domination. (We must assume that $n \geqslant 2$ in order that $\pi_{1}\left(K^{H}\right) \cong \pi_{1}\left(X^{H}\right)$ for every $H$.) If $H$ is a subgroup of $G$, then the inclusion $K^{H} \hookrightarrow X^{H}$ is an $N H / H$-domination. Let $p_{H}: \tilde{X}^{H} \rightarrow X^{H}$ be the universal cover. The spaces $\tilde{K}^{H}$ and $\tilde{X}^{H}$ are $\widetilde{N H / H}=\pi_{1}\left(X^{H}\right) x_{\alpha} N H / H$-complexes by (2.4), the cellular chain complexes $C\left(\tilde{K}^{H}\right)$ and $C\left(\tilde{X}^{H}\right)$ are $\mathbf{Z}(N H / H)$-permutation complexes by (3.1), and the inclusion $C\left(K^{H}\right) \hookrightarrow C\left(X^{H}\right)$ is a $\mathrm{Z}(N H / H)$ domination in which all maps are permutation maps. It easily follows that the induced map

$$
C\left(\widetilde{K^{H}}, p_{H}^{-1}\left(K^{>H}\right)\right) \hookrightarrow C\left(\widetilde{X^{H}}, p_{H}^{-1}\left(X^{>H}\right)\right)
$$

is a $\mathbf{Z}(\overparen{N H / H})$-domination for the free $\mathbf{Z}(\widetilde{N H / H})$-chain complexes. Since $C\left(\widetilde{K^{H}}, p_{H}^{-1}\left(K^{>H}\right)\right)$ is finite, $w\left(C\left(\widetilde{X^{H}}, p_{H}^{-1}\left(X^{>H}\right)\right)\right)$ is defined.

Definitions. Let $X$ satisfy the hypotheses of Theorem 1.

2. The relative Wall invariant of $X$ with respect to $H$ is defined by

$$
w_{H}(X) \equiv w\left(C\left(\widetilde{X^{H}}, p_{H}^{-1}\left(X^{>H}\right)\right)\right) \in \tilde{K}_{0}(\mathbf{Z}(\widetilde{N H / H})) .
$$

3. Define $E_{1}(X, G)=\Sigma_{H<G} w_{H}(X) \in \Sigma_{H<G} \tilde{K}_{0}(\widetilde{Z}(\widetilde{N H / H}))$.

Definition 4. Let $K$ and $X$ be $G$-complexes. Let $\varphi: K \rightarrow X$ be a $G$-map. The map $\varphi$ is called a $G$-homology-equivalence if

$$
\varphi_{*}^{H}: H\left(q^{-1}\left(K^{H}\right)\right) \rightarrow H\left(p^{-1}\left(X^{H}\right)\right)
$$

is an isomorphism for each subgroup $H$ of $G$, where $q: \hat{K} \rightarrow K$ is the pullback via $\varphi$ of the universal cover of $X$.

The invariant for $G$-equivalence used quotients of the chain complexes $C\left(\widetilde{X^{H}}\right)$. For $G$-homology-equivalence, the invariant will be defined using

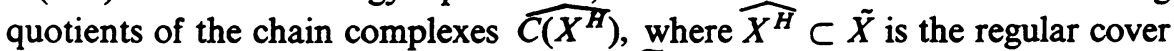
of $X^{H}$ which contains the fixed basepoint $\overline{e^{0}} \in p^{-1}\left(X^{G}\right)$.

Assume as before that $X$ is of finite type and the inclusion $K \hookrightarrow X$, where $K=X^{n}$ and $n \geqslant 2$, is a $G$-domination. Let $i_{H}: X^{H} \hookrightarrow X$ be the inclusion and 
let $\pi_{1}\left(i_{H}\right): \pi_{1}\left(X^{H}\right) \rightarrow \pi_{1}(X)$ be the induced homomorphism. Since im $\left(\pi_{1}\left(i_{H}\right)\right)$ $\subseteq \pi_{1}(X)$ has a trivial $H$-action, there is an induced action of $N H / H$ on $\operatorname{im}\left(\pi_{1}\left(i_{H}\right)\right)$. Hence by (2.4), $\widehat{X}^{H}$ is an $\widehat{N H / H}=\operatorname{im} \pi_{1}\left(i_{H}\right) x_{\alpha} N H / H$-complex. Analogous to the discussion above, the inclusion

$$
C\left(\widehat{K^{H}}, p^{-1}\left(K^{>H}\right) \cap \widehat{K^{H}}\right) \hookrightarrow C\left(\widehat{X^{H}}, p^{-1}\left(X^{>H}\right) \cap \widehat{X^{H}}\right)
$$

is a $\mathbf{Z}(\widehat{N H / H})$-domination for the free $\mathbf{Z}(\widehat{N H / H})$-complexes. Hence, the invariant $w\left(C\left(\widehat{X^{H}}, p^{-1}\left(X^{>H}\right) \cap \widehat{X^{H}}\right)\right)$ is defined.

Definition 5. Let $X$ satisfy the hypotheses of Theorem 1 . We define

$$
\begin{aligned}
E_{2}(X, G) & =\sum_{H<G} w\left(C\left(\widehat{X^{H}}, p^{-1}\left(X^{>H}\right) \cap \widehat{X^{H}}\right)\right) \\
& \in \sum_{H<G} \oplus \tilde{K}_{0}(\widehat{\mathbf{Z}(N H / H)}) .
\end{aligned}
$$

The invariants $E_{1}(X, G)$ and $E_{2}(X, G)$ are related.

Lemma 6. Let $X$ satisfy the hypotheses of Theorem 1. Then

(i) There is a homomorphism $\sigma_{H}: \widehat{N H / H} \rightarrow \widehat{N H / H}$, and therefore an induced homomorphism

$$
\sigma_{*}=\sum_{H<G} \sigma_{H_{*}}: \sum_{H<G} \bigoplus \tilde{K}_{0}(\mathrm{Z}(\overparen{N H / H})) \rightarrow \sum_{H<G} \bigoplus \tilde{K}_{0}(\mathbf{Z}(\widehat{N H / H})) .
$$

(ii) The invariants $E_{1}(X, G)$ and $E_{2}(X, G)$ satisfy the relation

$$
E_{2}(X, G)=\sigma_{*} E_{1}(X, G) \text {. }
$$

In particular, if each $\pi_{1}\left(i_{H}\right)$ is an isomorphism then $E_{2}(X, G)=E_{1}(X, G)$.

Proof. (i) The homomorphism $\sigma_{H}$ is defined by $(\gamma, g) \mapsto\left(\pi_{1}\left(i_{H}\right) \gamma, g\right), \gamma \in$ $\pi_{1}\left(X^{H}\right)$ and $g \in N H / H$.

(ii) By a relative form of (3.8)

$$
C\left(\widehat{X^{H}}, p^{-1}\left(X^{>H}\right) \cap \widehat{X^{H}}\right) \cong \mathbf{Z}(\widehat{N H / H}) \underset{\mathbf{Z}(\widehat{N H / H})}{\otimes} C\left(\widetilde{X^{H}}, p_{H}^{-1}\left(X^{>H}\right)\right) \text {. }
$$

Hence $w\left(C\left(\widehat{X^{H}}, p^{-1}\left(X^{>H}\right) \cap \widehat{X^{H}}\right)\right)=\sigma_{H_{0}} w_{H}(X)$. By summing over all subgroups $H$ we obtain the desired result.

It is clear that if $\varphi: K \rightarrow X$ is a $G$-equivalence then it is a $G$-homologyequivalence as well. In the context above, $E_{1}(X, G)=0$ implies $E_{2}(X, G)=$ 0 . At the end of this section we will give an example of a $G$-complex $X$ with the property that $E_{1}(X, G) \neq 0$ but $E_{2}(X, G)=0$, which implies that we can construct a $G$-homology-equivalence between $X$ and a finite complex when a $G$-equivalence does not exist.

Also note that since $g \in G$ is a homeomorphism it induces isomorphisms between projective class groups and $g_{*} w_{H}(X)=w_{g g^{-1}}(X)$ and $g_{*} \sigma_{*} w_{H}(X)$ $=\sigma_{*} w_{g g^{-1}}(X)$. We could have defined both invariants as sums over conjugacy classes of subgroups instead of as sums over all subgroups. 
Proof of Theorem 1 (i). The element $E_{1}(X, G)$ is an invariant of $G$ homotopy type since each $w_{H}(X)$ is an invariant of $\mathrm{Z}(\widehat{N H / H})$-chain homotopy type.

To prove the condition is necessary, suppose that $\varphi: K \rightarrow X$ is a $G$-equivalence. There is an induced $\mathrm{Z}(\widehat{N H / H})$-chain equivalence

$$
\varphi_{\sharp}^{H}: C\left(\widetilde{K^{H}}, q_{H}^{-1}\left(K^{>H}\right)\right) \rightarrow C\left(\widetilde{X^{H}}, p_{H}^{-1}\left(X^{>H}\right)\right),
$$

where $q_{H}: \widetilde{K^{H}} \rightarrow K^{H}$ is the universal cover.

Thus, $w_{H}(X)=0$ for each $H$. By definition $E_{1}(X, G)=0$.

To prove the sufficiency we first assume as above that $X$ is a $G$-complex of finite type which is $G$-dominated by its $n$-skeleton, $K$, and that $n \geqslant 2$. By induction, we assume that $K$ is an at most $(n+1)$-dimensional subcomplex of $X$ and we assume that the inclusion map $i_{H^{\prime}}: K^{H^{\prime}} \hookrightarrow X^{H^{\prime}}$ is a homotopy equivalence for all subgroups $H^{\prime}$ such that $\left|H^{\prime}\right|>|H|$. We let $H$ represent a conjugacy class of subgroups.

Consider the short exact sequence of $\mathbf{Z}(\widetilde{N H / H})$-complexes

$$
\begin{aligned}
C\left(p_{H}^{-1}\left(X^{>H}\right), p_{H}^{-1}\left(K^{>H}\right)\right) & \rightarrow C\left(\widetilde{X^{H}}, \widetilde{K^{H}}\right) \\
& \rightarrow C\left(\widetilde{X^{H}}, p_{H}^{-1}\left(X^{>H}\right) \cup \widetilde{K^{H}}\right) .
\end{aligned}
$$

By a relative form of (3.9),

$$
C\left(p_{H}^{-1}\left(X^{>H}\right), p_{H}^{-1}\left(K^{>H}\right)\right) \cong \mathbf{Z}(\widetilde{N H / H}) \underset{R}{\otimes} C\left(\widetilde{X^{>H}}, \widetilde{K^{>H}}\right)
$$

where $R=\mathbf{Z}\left(\pi_{1}\left(X^{>H}\right) x_{\alpha} N H / H\right)$. By the inductive hypothesis the inclusion $i_{>H}: K^{>H} \hookrightarrow X^{>H}$ is a homotopy equivalence. Then $C\left(\widetilde{X^{>H}}, \widetilde{K^{>H}}\right)$ is acyclic and, therefore, by the universal coefficient theorem, the complex

$$
C\left(p_{H}^{-1}\left(X^{>H}\right), p_{H}^{-1}\left(K^{>H}\right)\right)
$$

is acyclic. Thus, $H\left(\widetilde{X^{H}}, \widetilde{K^{H}}\right) \cong H\left(\widetilde{X^{H}}, p_{H}^{-1}\left(X^{>H}\right) \cup \widetilde{K^{H}}\right)$.

By assumption, the only nonzero homology group for either complex is in dimension $(n+1)$. Since $w_{H}(X)=0$ by the isomorphism above

$$
\left[H_{n+1}\left(\widetilde{X^{H}}, \widetilde{K^{H}}\right)\right]=0 \in \tilde{K}_{0}(\mathbf{Z}(\widetilde{N H / H})) \text {. }
$$

Thus, there exist finitely-generated free $\mathrm{Z}(\overparen{N H / H})$-modules, $F_{1}$ and $F_{2}$, such that

$$
F_{1} \cong H_{n+1}\left(\widetilde{X^{H}}, \widetilde{K^{H}}\right) \oplus F_{2} .
$$

In the following construction we will attach a finite number of cells to the subspace $K^{H}$ and to its homeomorphic copies $K^{g H^{-1}}, g \in G$. We then replace $X$ by a $G$-equivalent space of finite-type with an $(n+1)$-subcomplex $L$ satisfying: $i_{H^{\prime}}: L^{H^{\prime}} \hookrightarrow X^{H^{\prime}}$ is a homotopy equivalence for all subgroups $H^{\prime}$ such that either $\left|H^{\prime}\right|>|H|$ or $H^{\prime}$ is conjugate to $H$. 
Step 1. We define a complex $K^{\prime}=K \vee\left(V_{(i, g)} S_{(i, g)}^{n}\right)$ where $\{i\}$ represents a finite set of generators of $F_{2}$, and $g$ ranges over some set of coset representatives of $G / H$. We extend the action of $G$ to $K^{\prime}$ by defining $g^{\prime} S_{(i, g)}^{n} \equiv S_{(i, g)}^{n}$, where $g$ is a representative of $G / H$ and $g^{\prime} \in G$.

Define a $G$-map $\varphi^{\prime}: K^{\prime} \rightarrow X$ by $\left.\varphi^{\prime}\right|_{K}=$ inclusion and $\left.\varphi^{\prime}\right|_{S_{(i, s)}^{n}}=x_{0}$ where $x_{0}$ is the basepoint in $X$. Then $\varphi^{\prime}$ is clearly a $G$-domination extending the inclusion. By (4.1) we can replace $X$ by a $G$-equivalent space $X^{\prime}$ of finite type in such a way that $K^{\prime H}$ is a subcomplex of $X^{\prime H}$. For this new space we have the short exact sequence

$$
H_{n+1}\left(\widetilde{X^{\prime H}}, \widetilde{K^{H}}\right)>H_{n+1}\left(\widetilde{X^{\prime H}}, \widetilde{K^{\prime H}}\right) \rightarrow H_{n}\left(\widetilde{K^{\prime H}}, \widetilde{K^{H}}\right)
$$

which splits. Since the first term is clearly isomorphic to $H_{n+1}\left(\widetilde{X^{H}}, \widetilde{K^{H}}\right)$ we have the direct sum decomposition

$$
\begin{aligned}
H_{n+1}\left(\widetilde{X^{\prime H}}, \widetilde{K^{\prime H}}\right) & \cong H_{n+1}\left(\widetilde{X^{H}}, \widetilde{K^{H}}\right) \oplus H_{n}\left(\widetilde{K^{\prime H}}, \widetilde{K^{H}}\right) \\
& \cong H_{n+1}\left(\widetilde{X^{H}}, \widetilde{K^{H}}\right) \oplus F_{2} \cong F_{1} .
\end{aligned}
$$

Step 2. For each generator $t$ of $F_{1}$ associate an element $\sigma_{t} \in$ $\pi_{n+1}\left(X^{\prime H}, K^{\prime H}\right)$ using the following sequence of isomorphisms

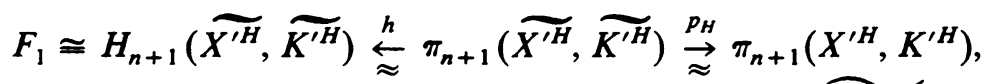

where $h$ represents the Hurewicz map. (These are all $\mathbf{Z}(\widetilde{N H / H})$-maps by functorial properties.) Let $\left(\beta_{t}, \gamma_{t}\right) \in \sigma_{t}$.

Define a complex $L$ by $L=K^{\prime} \cup_{(t, g)} e_{(t, g)}^{n+1}$ where the $(n+1)$-cell $e_{(t, g)}^{n+1}$ has attaching map $g \circ \gamma_{t}, g$ ranges over representatives of $G / H$, and $t$ ranges over generators of $F_{1}$. (This definition is independent of the choice of representatives in $G / H$ since $H$ fixes $K^{\prime H}$.) We extend the $G$-action on $K^{\prime}$ to $L$ by defining $g^{\prime} e_{(t, g)}^{n+1}=e_{\left(t, g^{\prime} g\right)}^{n+1}$. The map $\psi: L \rightarrow X^{\prime}$, extending the inclusion on $K^{\prime}$ by setting $\left.\psi\right|_{e_{(t, s)}^{n+1)}}=g \circ \beta_{l}$, is a $G$-dominating map.

By construction, the Hurewicz theorem and Whitehead's theorem, $\psi^{H}$ is a homotopy equivalence. Likewise, since each $g$ is a homeomorphism, it follows that $\psi^{8 \mathrm{Hg}^{-1}}$ is a homotopy equivalence. Since the fixed-point sets for $H^{\prime}$, where $\left|H^{\prime}\right|>|H|$, were not changed in the replacement of $X$ by $X^{\prime}$ in Step 1, then $\psi^{H^{\prime}}=i_{H^{\prime}}: L^{H^{\prime}} \hookrightarrow X^{\prime H^{\prime}}$ is a homotopy equivalence.

Finally, by (4.1) we can now assume that $L$ is a subcomplex of $X$, and our induction step is complete.

The induction will end in a finite number of steps since $G$ is finite. When $H=1$ Steps 1 and 2 and (4.4) imply that $\psi: L \rightarrow X$, is a $G$-equivalence.

Proof of Theorem 1 (ii). It is clear that the element $E_{2}(X, G)$ is an invariant of $G$-homotopy type.

To prove necessity, suppose that $\varphi: K \rightarrow X$ is a $G$-2-connected, $G$ homology-equivalence, where $K$ is a finite $G$-complex. Let $H$ be a subgroup of $G$. Considering $X$ as an $N H$-complex, by (2.5) and (3.9) 


$$
C\left(p^{-1}\left(X^{H}\right)\right) \cong \mathbf{Z}(\widetilde{N H}) \underset{\mathrm{Z}(\stackrel{\otimes}{N H})}{\otimes} C\left(\widehat{X^{H}}\right)
$$

where $\widetilde{N H}=\pi_{1}(X) x_{\alpha} \widehat{N H}$ and $N H=\operatorname{im}\left(\pi_{1}\left(i_{H}\right)\right) x_{\alpha} N H$. Since $\mathrm{Z}(\widetilde{N H})$ is a free $\mathbf{Z}(\widehat{N H})$-module, the universal coefficient theorem implies that

$$
H\left(p^{-1}\left(X^{H}\right)\right) \cong \mathbf{Z}(\widetilde{N H}) \underset{\mathbf{Z}(\widehat{N H})}{\otimes} H\left(\widehat{X^{H}}\right) .
$$

A similar argument shows that $H\left(p^{-1}\left(X^{>H}\right)\right) \cong \mathbf{Z}(\widetilde{N H}) \otimes_{\mathrm{Z}(\widehat{N H})} H\left(\widehat{\left(X^{>H}\right)}\right)$.

By replacing $X$ by the mapping cylinder $M(\varphi)$ we can consider $K^{H}$ as a subspace of $X^{H}$. Applying the above analysis we see that

$$
0=H\left(p^{-1}\left(X^{H}\right), p^{-1}\left(K^{H}\right)\right) \simeq \mathrm{Z}(\widetilde{N H}) \underset{\mathrm{Z}(\widehat{N H})}{\otimes} H\left(\widehat{X^{H}}, \widehat{K^{H}}\right) .
$$

Since the last term is a direct sum, we have $H\left(\widehat{X^{H}}, \widehat{K^{H}}\right)=0$. Similarly, $H\left(\widehat{X^{>H}}, \widehat{K^{>H}}\right)$ is zero.

Since $\widehat{X^{H}} \cap p^{-1}\left(X^{>H}\right)$ is a union of components each homeomorphic to $\widehat{X>H}$, it follows that

$$
H\left(\widehat{K^{H}}, p^{-1}\left(K^{>H}\right) \cap \widehat{K^{H}}\right) \cong H\left(\widehat{X^{H}}, p^{-1}\left(X^{>H}\right) \cap \widehat{X^{H}}\right)
$$

as $\mathbf{Z} \widehat{N H})$-modules and as $\mathbf{Z}(\widehat{N H / H})=\mathbf{Z}\left(\operatorname{im}\left(\pi_{1}\left(i_{H}\right)\right) x_{\alpha} N H / H\right)$-modules. Since these chain complexes are $\mathbf{Z}(\overline{N H / H})$-free they are also chain equivalent. Hence, $w\left(C\left(\widehat{X^{H}}, p^{-1}\left(X^{>H}\right) \cap \widehat{X^{H}}\right)\right)=0$. It then follows that $E_{2}(X, G)$ $=0$.

Now assume that $E_{2}(X, G)=0$. Assume that $X$ is of finite type and the inclusion $K \hookrightarrow X$ is a $G$-domination, where $K=X^{n}$ and $n \geqslant 2$. Since the proof that $X$ is $G$-homology-equivalent to a finite complex is similar in part to the proof of $G$-equivalence from part (i) of this theorem, we will omit many details.

The induction hypothesis states: $K$ is a subcomplex of $X$ of dimension (at

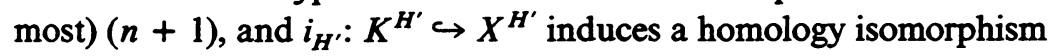

$$
H\left(p^{-1}\left(K^{H^{\prime}}\right)\right) \cong H\left(p^{-1}\left(X^{H^{\prime}}\right)\right)
$$

for all subgroups $H^{\prime}$ such that $\left|H^{\prime}\right|>|H|$. We let $H$ represent a conjugacy class of subgroups.

From our inductive hypothesis and the previous discussion,

$$
C\left(p^{-1}\left(X^{>H}\right) \cap \widehat{X^{H}}, p^{-1}\left(K^{>H}\right) \cap \widehat{K^{H}}\right)
$$

is acyclic. Thus, $H\left(\widehat{X^{H}}, \widehat{K^{H}}\right) \cong H\left(\widehat{X^{H}}, \widehat{K^{H}} \cup\left(p^{-1}\left(X^{>H}\right) \cap \widehat{X^{H}}\right)\right)$. Since $E_{2}(X, G)=0$ there exist f.g. free $\mathbf{Z}(\overline{N H / H})$-modules, $F_{1}$ and $F_{2}$, such that $F_{1} \cong H_{n+1}\left(\widehat{X^{H}}, \widehat{K^{H}}\right) \oplus F_{2}$.

In our construction we attach orbits of cells to $U_{g} K^{g H_{g}^{-1}}$. We then replace $X$ by a $G$-equivalent space of finite type containing an $(n+1)$-subcomplex $L$ 
satisfying: the inclusion induces isomorphisms

$$
H\left(p^{-1}\left(L^{H^{\prime}}\right)\right) \cong H\left(p^{-1}\left(X^{H^{\prime}}\right)\right)
$$

for $H^{\prime}$ conjugate to $H$ or $\left|H^{\prime}\right|>|H|$.

Step 1. Follow Step 1 of part (i). The last line becomes $H_{n+1}\left(\widetilde{X^{\prime H}}, \widetilde{K^{\prime H}}\right) \cong$ $H_{n+1}\left(\widetilde{X^{H}}, \widetilde{K^{H}}\right) \oplus F$, where $F$ is $\mathbf{Z}(\widehat{N H / H})$-free and has the same rank as $F_{2}$. But now applying $\left[\mathrm{Z}(\widehat{N H / H}) \otimes_{\mathbf{Z}(N H / H)}\right]$ to both sides yields $H_{n+1}\left(\widehat{X^{\prime H}}, \widehat{K^{\prime H}}\right)$ $\simeq F_{1}$.

Step 2. The Hurewicz homomorphism

$$
h: \pi_{n+1}\left(\widehat{X^{\prime H}}, \widehat{K^{\prime H}}\right) \rightarrow H_{n+1}\left(\widehat{X^{\prime H}}, \widehat{K^{\prime H}}\right)
$$

is onto by the Hurewicz theorem. Hence there is a $\mathbf{Z}(\widehat{N H / H})$ homomorphism $\sigma: F_{1} \rightarrow \pi_{n+1}\left(X^{\prime H}, K^{\prime H}\right)$. Use this correspondence in Step 2 of part (i) to construct $L$ and $\psi: L \rightarrow X$. Assume $L \subset X$ by (4.1). By construction we have the required homology isomorphisms.

EXAMple 7. We will construct a $\mathbf{Z}_{2}$-complex $X$ of finite type which is $\mathbf{Z}_{2}$-dominated by its 2-skeleton. For this complex $E_{1}\left(X, \mathbf{Z}_{2}\right) \neq 0$ but $E_{2}\left(X, \mathbf{Z}_{2}\right)$ $=0$. Thus $X$ is not $Z_{2}$-equivalent to a finite complex, but there does exist $\psi$ : $L \rightarrow X$ where $L$ is finite and $\psi$ is a $G$-homology-equivalence.

The group $\tilde{K}_{0}\left(\mathbf{Z}\left(\mathbf{Z}_{23}\right)\right) \cong \mathbf{Z}_{3}\left[12\right.$, p. 30]. Let $w_{0}$ be a generator. Define a 2-complex $K=S^{1} \cup_{23} e^{2}$, by adjoining a 2-cell to $S^{1}$ by a map of degree 23. Then $\pi_{1}(K) \cong Z_{23}$. By [15, Theorem F] there exists a complex $Y$ of finite type such that $K \subset Y^{2}, Y^{1}=K^{1}, Y^{2}$ dominates $Y$ and $w(Y)=w(C(\tilde{Y}))=$ $w_{0} \in \tilde{K}_{0}\left(\mathbf{Z}\left(\mathbf{Z}_{23}\right)\right)$. The space $Y$ will be $X^{\mathbf{Z}_{2}}$ in our example.

Let $X=Y \cup e_{1}^{2} \cup e_{2}^{2}$ where the 2-cells are attached via 1: $S^{1} \rightarrow S^{1} \subset Y$. Clearly $\pi_{1}(X)=1$. Let $Z_{2}$ act on $X$ by switching the 2-cells.

Let $j: Y^{2} \hookrightarrow Y$ be the inclusion, let $s: Y \rightarrow Y^{2}$ be a right homotopy inverse such that $\left.s\right|_{Y^{1}}$ is the identity and let $F: j s \cong 1_{Y}\left(\right.$ rel $\left.Y^{1}\right)$ be a homotopy. We will extend these to $Z_{2}$-maps as follows: extend $s$ to $s^{\prime}: X \rightarrow X^{2}$ be defining it to be the identity on the 2-cells; extend $F$ to $F^{\prime}: X \times I \rightarrow X$ by $(x, t) \mapsto x$, $x \in e_{1}^{2} \cup e_{2}^{2}$ and $t \in I$. Let $i: X^{2} \hookrightarrow X$. Then $s^{\prime}$ is a right $\mathbf{Z}_{2}$-homotopy inverse of $i$. Therefore, $X^{2} \mathbf{Z}_{2}$-dominates $X$.

The invariant

$$
E_{1}\left(X, \mathbf{Z}_{2}\right)=w_{\mathbf{Z}_{2}}(X)+w_{1}(X)=w(Y)+w_{1}(X)=w_{0} \neq 0 .
$$

$\left(w_{1}(X)=w(C(X, Y)) \in \tilde{K}_{0}\left(\mathbf{Z}\left(\mathbf{Z}_{2}\right)\right)=0\right.$. $)$ The invariant $E_{2}\left(X, \mathbf{Z}_{2}\right)=$ $w\left(C\left(X^{\mathbf{Z}_{2}}\right)\right)+w\left(C\left(X, X^{\mathbf{Z}_{2}}\right)\right) \in \tilde{K}_{0}(\mathbf{Z}) \oplus \tilde{K}_{0}\left(\mathbf{Z}\left(\mathbf{Z}_{2}\right)\right)=0$. Hence, $E_{2}\left(X, \mathbf{Z}_{2}\right)=$ 0 .

The results of this section suggest a related problem: Let $G$ be a finite group. Define a Wall invariant in the category of $R G$-permutation complexes and maps. We note here that such an invariant can be defined, but we omit the details. 
6. Further results. Let $\varphi: K \rightarrow X$ be a $G$-dominating map where $K$ is a finite $G$-complex. In the previous sections we have studied the problems of replacing $\varphi$ by either a $G$-homotopy equivalence or a $G$-homology equivalence $\psi$ : $L \rightarrow X$ where $L$ is a finite $G$-complex. Since we can always assume that a $G$-domination is G-2-connected, a positive solution to either of these problems would show that it is possible to replace $\varphi$ by a $G$-map $\psi: L \rightarrow X$ which is an ordinary homotopy equivalence.

It is the object of this section to investigate the problem of the existence of a $G$-homology equivalence further, with the additional necessary assumption that there exists a $G$-map which is an ordinary homotopy equivalence. We note that, by [8, Theorem 1.1] any finite complex whose reduced homology with $\mathbf{Z}_{p}$-coefficients is trivial, can be the fixed-point set of finite contractible $\mathbf{Z}_{p}$-complex. Therefore, a $G$-map which is an ordinary homotopy equivalence (in this case a contraction) need not be a $G$-homology-equivalence.

Our main results are Theorems 1 and 2 .

THEOREM 1. Let $X$ be a $G$-complex such that $X^{H}$ is connected and $X^{G} \neq \varnothing$. Suppose that $\pi_{1}(X)$ is finite and that either $G$ acts semi-freely on $X$ or $G$ is solvable. Let $\varphi: K \rightarrow X$ be a $G$-dominating map where $K$ is a finite $G$-complex, the dimension of $K$ is $n$, and $n \geqslant 2$. Then the following statements are equivalent:

(i) The invariant $E_{2}(X, G)=0$.

(ii) There exists a G-n-connected, G-dominating map $\psi: L \rightarrow X$ where $L$ is a finite complex and $\operatorname{dim}(L)=n+1$ such that $\psi$ is an ordinary homotopy equivalence.

In Theorem 2 we let $\widehat{\hat{\varphi}^{G}}: \widehat{K^{G}} \rightarrow \widehat{X^{G}}$ be the restriction of $\tilde{\varphi}: \tilde{K} \rightarrow \tilde{X}$ to the regular cover of $K^{G}$ in $\tilde{K}$. Also, we let $\tilde{\varphi}^{\sigma}: \widehat{K}^{G} \rightarrow X^{G}$ be the restriction to the complete inverse image of $K^{G}$ in $\tilde{K}$. The inclusion of $X^{G}$ into $X$ is denoted by $i_{G}$.

TheOREM 2. Let $X$ be a $G$-complex and let $G$ act semi-freely on $X$. Suppose that $X^{G}$ is connected and not empty. Let $\varphi: K \rightarrow X$ be a G-2-connected $G$-domination which is also a homotopy equivalence.

(i) If each $H_{i}\left(M\left(\widehat{\varphi^{G}}\right), \widehat{K^{G}}\right)$ has a finite $\mathrm{Z}\left(\mathrm{im}\left(\pi_{1}\left(i_{G}\right)\right)\right)$-free resolution of length 2, then $E_{2}(X, G)=0$.

(ii) If each $H_{i}\left(M \widehat{\left.\varphi^{\sigma}\right)}, \widehat{\left.K^{\sigma}\right)}\right.$ has a finite $\mathrm{Z} \tilde{G}$ resolution of length 2 , then $E_{2}(X, G)=w\left(C \widehat{\left(X^{G}\right)}\right)$. In addition, if $X$ is simply connected then $E_{2}(X, G)=$ 0.

Some special cases of Theorem 2 are of interest. 
Corollary 3. Let $X, G$ and $\varphi: K \rightarrow X$ be defined as in Theorem 2. Suppose that $\pi_{1}(X)$ is finite. Then the finite resolutions of $H_{i}\left(M\left(\varphi^{G}\right), \widehat{K^{G}}\right)$ and $H_{i}\left(M\left(\varphi^{G}\right), K^{G}\right)$ in parts (i) and (ii) may be taken to be of any finite length.

Proof. It is sufficient to show that either module has a finite resolution of length 2.

Let $\pi$ be a finite group and $F_{n} \rightarrow F_{n-1} \rightarrow \cdots \rightarrow F_{0} \rightarrow M$ be a finite $\mathrm{Z}_{\pi}$-free resolution of $M$. There exists a finite projective resolution of $M$ of length $2, P_{1} \rightarrow P_{0} \rightarrow M$, by [13, Theorem 4.12]. We may assume that $P_{0}$ is free. There exists a chain map $\varphi: P \rightarrow F$ such that the algebraic mapping cone $M(\varphi)$ is acyclic. By Schanuel's Lemma [1, p. 36] applied to $M(\varphi)$ and the zero complex, it follows that $P_{1}$ is stably-free. Thus, there exist f.g. free modules $F_{1}^{\prime}$ and $F_{2}^{\prime}$ such that $F_{2}^{\prime} \cong F_{1}^{\prime} \oplus P_{1}$. Then $F_{1}^{\prime} \oplus P_{1}^{\prime} \rightarrow F_{1}^{\prime} \oplus$ $P_{0} \rightarrow M$ is the desired resolution.

COROLLARY 4. Let $X$ be a $\mathbf{Z}_{n}$-complex which is simply connected. Let $K$ be a finite $\mathbf{Z}_{n}$-complex and $\varphi: K \rightarrow X$ be a $\mathbf{Z}_{n}$-dominating 2-connected map which is a homotopy equivalence. Suppose that $H\left(M\left(\varphi^{\mathrm{Z}_{n}}\right), K^{\mathrm{Z}_{n}}\right)$ is finite and prime to $n$. Then $X$ is $\mathbf{Z}_{n}$-homology equivalent to a finite complex.

Proof of Corollary 4. By [8, Lemma 1.1] each $H_{i}\left(M\left(\varphi^{\mathbf{Z}_{n}}\right), K^{\mathbf{Z}_{n}}\right)$ has a finite free $\mathbf{Z}\left(\mathbf{Z}_{n}\right)$-resolution of length 2 . The rest follows from Corollary 3 and (6.1).

The proofs of the main theorems will follow from Lemmas 5-7.

LEMMA 5. Let $G$ be a finite group. Then $G$ is solvable if and only if for each subgroup $H$ of $G$ there exists a maximal proper subgroup $M$ of $H$ which is normal in $H$.

Proof. Recall that a finite group is solvable if and only if the factor groups in a composition series are cyclic of prime order [7, p. 139].

Let $G$ be a solvable group. Since each subgroup of a solvable group is solvable, it suffices to prove that a solvable group has a maximal proper subgroup which is normal. Let $G=G_{0} \triangleright G_{1} \triangleright \cdots \triangleright G_{n}=1$ be a composition series for $G$. Then $G / G_{1}$ is cyclic of prime order and thus $G_{1}$ is maximal in $G$.

Suppose that $G$ is not solvable. Let $G \triangleright G_{1} \triangleright G_{2} \triangleright \cdots \triangleright G_{n}=1$ be a composition series for $G$. Then there exists an $i$ such that the order of $G_{i} / G_{i+1}$ is the form $p q$ where $p$ is prime and $q \neq 1$. Hence $G_{i} / G_{i+1}$ contains a subgroup of order $p$. It follows that although $G_{i+1}$ is maximal among the normal subgroups of $G_{i}$, it is not maximal among the subgroups of $G_{i}$.

LEMMA 6. Let $G$ be a finite group. Let $P$ be a finitely-generated projective $\mathrm{ZG-module,} \mathrm{and} T$ be a finitely-generated free abelian group with trivial $\mathrm{ZG}$-module structure. If $P \cong T$ as $\mathrm{ZG}$-modules, then $P=T=0$. 
Proof. Let $\hat{H}(G, M)$ represent the complete derived homology and cohomology sequence of $G$ with coefficients in the $Z G$-module $M$ [4, Chapter XII]. For a projective $P, \hat{H}(G, P)=0[4$, p. 236]. If the trivial module $T \neq 0$, then there are finite direct sums $T=\Sigma \oplus \mathbf{Z}$ and $H(G, T) \cong \Sigma \oplus H(G, \mathbf{Z}) \neq$ 0 by [4, p. 237] and thus $P$ and $T$ are not isomorphic as $Z G$-modules.

LEMMA 7. Let $\boldsymbol{P}$ be a finitely-generated projective $\mathbf{Z} \pi$-module where $\pi$ is a finite group. If $\mathrm{Z} \otimes_{\mathrm{Z} \pi} P=0$, then $P=0$.

Proof. Suppose $P \neq 0$. By a theorem of Swan $\left[5\right.$, p. 556] $P \simeq F \oplus P_{0}$ where $F$ is a free $\mathrm{Z} \pi$-module and $P_{0}$ is a projective ideal in $Z \pi$. Since the tensor product commutes with direct sums it follows that if $\mathbf{Z} \otimes_{\mathrm{Z}_{\pi}} P=0$ then $F=0$ and $P=P_{0}$.

By [5, p. 557] $P \otimes_{\mathrm{Z}} \mathrm{Q} \cong \mathrm{Z} \pi \otimes_{\mathrm{z}} \mathrm{Q} \cong \mathbf{Q} \pi$ where $\mathrm{Q}$ is the field of rational numbers. Now we have

$$
0=(\mathbf{Z} \underset{\mathbf{Z} \pi}{\otimes} P) \underset{\mathbf{Z}}{\otimes} \mathbf{Q} \cong \mathbf{Z} \underset{\mathbf{Z} \pi}{\otimes}(P \underset{\mathbf{Z}}{\otimes} \mathbf{Q}) \cong \mathbf{Z} \underset{\mathbf{Z} \pi}{\otimes} \mathbf{Q} \pi \geq \mathbf{Z} \underset{\mathbf{Z} \pi}{\otimes} \mathbf{Z} \pi \cong \mathbf{Z} .
$$

Thus, we have a contradiction.

Proof OF THEOREM 1. (i) $\Rightarrow$ (ii) by the proof of (5.1). (ii) $\Rightarrow$ (i): By (4.1) we assume that $\varphi: K \rightarrow X$ is inclusion, $K=X^{n}$ and $n \geqslant 2$.

Suppose first that $G$ is solvable.

By assumption $C(\tilde{X}, \tilde{K})$ is acyclic. Suppose that for all $H^{\prime}$ such that $\left|H^{\prime}\right|<|H|, C\left(\widehat{X^{H^{\prime}}}, \widehat{\left.K^{H^{\prime}}\right)}\right.$ and $C\left(X^{H^{\prime}}, K^{H^{\prime}}\right)$ are acyclic, and let $H$ be a subgroup of $G$. By Lemma 5 there exists a proper normal subgroup $M$ of $H$ such that $H / M$ is a cyclic group of prime order. Thus $H / M$ acts semi-freely on $X^{M}$. Note that its fixed-point set is $\left(X^{M}\right)^{H / M}=X^{H}$. To simplify notation let $\overparen{X^{H}}=p^{-1}\left(X^{H}\right) \cap \widehat{X^{M}}$.

(1) Consider the short exact sequence of $\mathbf{Z}\left(\operatorname{im}\left(\pi_{1}\left(i_{M}\right)\right)\right.$-modules. (We ignore the action of $H / M$ on the spaces in this step.)

$$
C\left(\widehat{X^{H}}, \widehat{K^{H}}\right) \longmapsto C\left(\widehat{X^{M}}, \widehat{K^{M}}\right) \rightarrow C\left(\widehat{X^{M}}, \widehat{X^{H}} \cup \widehat{K^{M}}\right) \text {. }
$$

By the inductive assumption $C\left(\widehat{X^{M}}, \widehat{K^{M}}\right)$ is acyclic. Thus $H_{i+1}\left(\widehat{X^{M}}, \widehat{X^{H}} \cup\right.$ $\left.\widehat{K^{M}}\right) \cong H_{i}\left(\widehat{X^{H}}, \widehat{K^{H}}\right)$ for all $i$. By assumption $\varphi^{H}$ is $(n-1)$-connected, and thus $H_{i}\left(\widehat{X^{H}}, \widehat{K^{H}}\right)=0=H_{i}\left(\widehat{X^{H}}, \widehat{K^{H}}\right)$ for $i<(n-1)$, where $n=\operatorname{dim}(K)$. Since $C\left(\widehat{K^{M}}, \widehat{K^{H}}\right)$ dominates $C\left(\widehat{X^{M}}, \widehat{X^{H}}\right)$,

$$
H_{i}\left(\widehat{X^{M}}, \widehat{X^{H}} \cup \widehat{K^{M}}\right)=0
$$

for $i>n+1$. Thus, the only nonzero groups are $H_{n+1}\left(\widehat{X^{M}}, \widehat{X^{H}} \cup \widehat{K^{M}}\right) \simeq$ $H_{n}\left(\widehat{\widehat{X^{H}}}, \widehat{K^{H}}\right)$.

The module $H_{n+1}\left(\widehat{X^{M}}, \widehat{X^{H}} \cup \widehat{K^{M}}\right)$ is finitely-generated $\mathbf{Z}\left(\operatorname{im}\left(\pi_{1}\left(i_{M}\right)\right)\right.$ projective. 
(2) By relative forms of (3.8) and (3.9) $C\left(X^{H}, K^{H}\right) \simeq \mathrm{Z} \otimes C\left(\widehat{X^{H}}, \widehat{K^{H}}\right)$, where we tensor over $\mathbf{Z}\left(\mathrm{im} \pi_{2}\left(i_{M}\right)\right)$. By Step 1 the only nonzero homology group of $C\left(\widehat{X^{H}}, \widehat{K^{H}}\right)$ is a f.g. projective. Hence, by the universal coefficient theorem, $H_{n}\left(X^{H}, K^{H}\right) \cong \mathrm{Z} \otimes H_{n}\left(\widehat{\widehat{X^{H}}}, \widehat{K^{H}}\right)$, which is a f.g. Z-free module.

(3) Consider the sequence of $\mathbf{Z}(H / M)$-complexes

$$
C\left(X^{H}, K^{H}\right) \rightarrow C\left(X^{M}, K^{M}\right) \rightarrow C\left(X^{M}, X^{H} \cup K^{M}\right) \text {. }
$$

By the inductive assumption and Step 2, the only nonzero groups are $H_{n+1}\left(X^{M}, X^{H} \cup K^{M}\right) \cong H_{n}\left(X^{H}, K^{H}\right)$. Since $C\left(K^{M}, K^{H}\right)$ dominates $C\left(X^{M}, X^{H}\right)$ as free $\mathbf{Z}(H / M)$-complexes, $H_{n+1}\left(X^{M}, X^{H} \cup K^{M}\right)$ is a finitelygenerated projective $\mathbf{Z}(H / M)$-module.

(4) Since the action of $H / M$ on $C\left(X^{H}, K^{H}\right)$ is necessarily trivial, Lemma 6 implies that

$$
H_{n+1}\left(X^{M}, X^{H} \cup K^{M}\right)=H_{n}\left(X^{H}, K^{H}\right)=0 .
$$

By Lemma 7, since

$$
H_{n}\left(X^{H}, K^{H}\right) \cong \mathrm{Z} \otimes H_{n}\left(\widehat{X^{H}}, \widehat{K^{H}}\right) \text { and } H_{n}\left(X^{H}, K^{H}\right)=0 \text {, }
$$

$H_{n}\left(\widehat{X^{H}}, \widehat{K^{H}}\right)=0$ and thus $H_{n+1}\left(\widehat{X^{M}}, \widehat{X^{H}} \cup \widehat{K^{M}}\right)=0$.

The induction step is complete.

Now suppose that $G$ acts semi-freely on $X$. Since we only need to consider $C\left(\tilde{X}, \overparen{X^{G}} \cup \tilde{K}\right)$ and $C\left(\overparen{X^{G}}, \widehat{K^{G}}\right)$ to check for $G$-homology equivalence, just consider $G$ and $M=1$ in the above induction step.

The proof of Theorem 1 can be applied more generally if we know something about the action of $G$ on $X$. Namely, if for each nontrivial subgroup $H$ of $G$ there exists a subgroup $M$ of $G$ such that $M$ is normal in $H$, $M \neq H$ and $H / M$ acts semi-freely on $X^{H}$, then the proof of Theorem 1 goes through. Lemma 5 shows that the proof cannot be extended to any larger class of subgroups without some extra conditions on the action.

The next lemma is used in the proof of Theorem 2.

LEMMA 8. Let $C$ be a free $R$-complex, and let $\varphi: D \rightarrow C$ be an $R$-dominating map, where $D$ is a finite free $R$-complex. Suppose that each $H_{i}(M(\varphi))$ has a finite free $R$-resolution of length 2 . Then $C$ is $R$-chain equivalent to a finite free $R$-complex.

Proof. We assume by induction that the map $\varphi: D \rightarrow C$ has the property that $H_{j}(M(\varphi))=0$ for $j \leqslant i$. To prove the lemma it is sufficient to construct a finite free chain complex $E$ and chain map $\psi: E \rightarrow C$ such that $H_{j}(M(\varphi))=0$ for $j<i+1$ and $H_{j}(M(\psi))=H_{j}(M(\varphi))$ for $j>i+1$. The construction will terminate in a finite number of steps since $\varphi$ is a domination and $D$ is finite.

The construction will be in two steps. Let $F^{\prime} \gg F \stackrel{h}{\rightarrow} H_{i+1}(M(\varphi))$ be a finite free resolution of $H_{i+1}(M(\varphi))$. 
(1) Use the map $h: F \rightarrow H_{i+1}(M(\varphi))$ and [3, Lemma 3] to construct a finite free complex $\bar{D}$ and map $\bar{\varphi}: \bar{D} \rightarrow C$. Then $H_{j}(M(\bar{\varphi})) \cong H_{j}(M(\varphi))$ for $j \neq i+1, i+2$. By construction, $H_{i+1}(M(\bar{\varphi}))=0$ and $H_{i+2}(M(\varphi)) \simeq$ $H_{i+2}(M(\varphi)) \oplus F^{\prime}$.

(2) Use the map $h^{\prime}: F^{\prime} \hookrightarrow H_{i+2}(M(\varphi)) \oplus F^{\prime} \cong H_{i+2}(M(\varphi))$ and [3, Lemma 3] to construct a finite complex $E$ and map $\psi: E \rightarrow C$ which satisfy the properties listed above.

Proof of Theorem 2. Since $G$ acts semi-freely on $X$,

$$
E_{2}(X, G)=w\left(C\left(\widehat{X^{G}}\right)\right)+w\left(C\left(\tilde{X}, p^{-1}\left(X^{G}\right)\right)\right.
$$

By assumption $C(M(\tilde{\varphi}), \tilde{K})$ is acyclic and

$$
H_{0}\left(M\left(\widehat{\varphi^{G}}\right), \widehat{K^{G}}\right)=H_{0}\left(M(\tilde{\varphi}), M\left(\widehat{\varphi^{G}}\right) \cup \tilde{K}\right)=0 .
$$

We also have the following isomorphisms:

$$
\begin{aligned}
H_{i+1}\left(M(\tilde{\varphi}), M\left(\widehat{\varphi^{G}}\right) \cup \tilde{K}\right) & \approx H_{i}\left(M\left(\widehat{\varphi^{G}}\right), p^{-1}\left(K^{G}\right)\right) \\
& \approx \mathbf{Z} \tilde{G} \otimes H_{i}\left(M\left(\widehat{\varphi^{G}}\right), \widehat{K^{G}}\right) .
\end{aligned}
$$

In the last term we tensor over $\mathbf{Z}\left(\operatorname{im} \pi_{1}\left(i_{G}\right)\right)$. Let $\varphi_{\#}^{G}: C\left(\widehat{K^{G}}\right) \rightarrow C\left(\widehat{X^{G}}\right)$ and $\varphi_{\#}: C\left(\tilde{K}, p^{-1}\left(K^{G}\right)\right) \rightarrow C\left(\tilde{X}, p^{-1}\left(X^{G}\right)\right)$ be induced chain maps, and let $M\left(\varphi_{\#}^{G}\right)$ and $M\left(\varphi_{\#}\right)$ be the algebraic mapping cones. Then $H\left(M\left(\varphi_{\#}^{G}\right)\right) \simeq$ $H\left(M\left(\widehat{\varphi^{G}}\right), \widehat{K^{G}}\right)$ and $H\left(M\left(\varphi_{\#}\right)\right) \simeq H\left(M(\tilde{\varphi}), M\left(\widehat{\varphi^{G}}\right) \cup \tilde{K}\right)$.

(i) By assumption and Lemma $8, w\left(C\left(\widetilde{X^{G}}\right)\right)=0$.

Let $F_{i P} \rightarrow F_{i 0} \rightarrow H_{i}\left(M\left(\varphi_{\#}^{G}\right)\right)$ be 'a finite $\mathbf{Z}\left(\mathrm{im}\left(\pi_{1}\left(i_{G}\right)\right)\right.$-resolution. By tensoring with $\mathbf{Z} \tilde{G}$ over $\mathbf{Z}\left(\operatorname{im}\left(\pi_{1}\left(i_{G}\right)\right)\right.$ we obtain an induced $\mathbf{Z} \tilde{G}$-resolution

$$
F_{i 1}^{\prime} \longrightarrow F_{i 0}^{\prime} \rightarrow H_{i+1}\left(M\left(\varphi_{\#}\right)\right) \text {. }
$$

Since $H_{0}\left(M\left(\varphi_{\#}\right)\right)=0$ we can apply Lemma 8 to obtain $w\left(C\left(\tilde{X}, p^{-1}\left(X^{G}\right)\right)\right)=$ 0.

(ii) By the assumption, the isomorphisms above and Lemma 8, $w\left(C\left(\tilde{X}, p^{-1}\left(X^{G}\right)\right)\right)=0$. Hence, $E_{2}(X, G)=w\left(C\left(\widehat{X^{G}}\right)\right)$.

If $X$ is simply-connected, then $w\left(C\left(\widehat{X^{G}}\right)\right)=w\left(C\left(X^{G}\right)\right) \in \tilde{K}_{0}(Z)=0$. Thus, $E_{2}(X, G)=0$.

\section{BIBLIOGRAPHY}

1. H. Bass, Algebraic K-theory, Benjamin, New York, 1968.

2. G. Bredon, Introduction to compact transformation groups, Academic Press, New York, 1972.

3. K. Brown, Homological criteria for finiteness, Comment. Math. Helv 50 (1975), 129-135.

4. H. Cartan and S. Eilenberg, Homological algebra, Princeton Univ. Press, Princeton, N. J., 1956.

5. C. Curtis and I. Reiner, Representation theory of finite groups and associative algebras, Wiley, New York, 1966.

6. S. M. Gersten, A product formula for Wall's obstruction, Amer. J. Math. 88 (1966), 337-346.

7. M. Hall, The theory of groups, Macmillan, New York, 1959. 
8. L. Jones, The converse to the fixed point theorem of P. A. Smith. I, Ann. of Math. 94 (1971), 52-68.

9. A. Lundell and S. Weingram, The topology of CW complexes, Van Nostrand Reinhold, New York, 1969.

10. T. Matumoto, On G-CW complexes and a theorem of J. H. C. Whitehead, J. Fac. Sci. Univ. Tokyo Sect. I A Math. 18 (1971), 363-374.

11. J. Milnor, Introduction to algebraic K-theory, Princeton Univ. Press, Princeton, N. J., 1971.

12. U Uses of the fundamental group, Colloquium Lectures, 73rd Summer Meeting of the Amer. Math. Soc., University of Wisconsin, 1968.

13. D. Rim, Modules over finite groups, Ann. of Math. 69 (1959), 700-712.

14. E. H. Spanier, Algebraic topology, McGraw-Hill, New York, 1966.

15. C. T. C. Wall, Finiteness conditions for CW complexes, Ann. of Math. 81 (1965), 55-69.

16. __ Finiteness conditions for CW complexes. II, Proc. Roy. Soc. London Ser. A 295 (1966), 129-139.

Departmient of Mathiematics, Fatrfietd Universtity, Fatrfield, ConNecticut 06430 